\title{
Estudo sobre a presença simultânea de dermatomicoses e diabetes em pacientes residentes em zona rural de um município do Estado do Paraná
}

\author{
Study on the simultaneous presence of dermatomycoses and diabetes in patients living in a rural \\ area from a municipality in the Paraná State
}

Estudio sobre la presencia simultánea de dermatomicosis y diabetes en pacientes residentes en una zona rural de un municipio del Estado de Paraná

Recebido: 01/07/2021 | Revisado: 09/07/2021 | Aceito: 13/07/2021 | Publicado: 23/07/2021

\author{
Waylla Albuquerque de Jesus \\ ORCID: https://orcid.org/0000-0003-3775-6832 \\ Faculdade Adventista Paranaense, Brasil \\ E-mail: w.albuquerque11@gmail.com \\ Camila Barros Galinari \\ ORCID: https://orcid.org/0000-0001-9728-8298 \\ Universidade Estadual de Maringá, Brasil \\ E-mail: camisgalinari@gmail.com \\ Glaucia Sayuri Arita \\ ORCID: https://orcid.org/0000-0001-9728-8298 \\ Universidade Estadual de Maringá, Brasil \\ E-mail: glauciasayuria@gmail.com \\ Valéria Aparecida Baquetti Mosca \\ ORCID: https://orcid.org/0000-0002-0651-290X \\ Universidade Estadual de Maringá, Brasil \\ E-mail: valeria.bmosca@gmail.com \\ Patrícia de Souza Bonfim de Mendonça \\ ORCID: https://orcid.org/0000-0002-0116-2189 \\ Universidade Estadual de Maringá, Brasil \\ E-mail: psbmendonca@gmail.com \\ Terezinha Inez Estivalet Svidzinski \\ ORCID: https://orcid.org/0000-0003-2525-9046 \\ Universidade Estadual de Maringá, Brasil \\ E-mail: tiesvidzinski@uem.br
}

\begin{abstract}
Resumo
Diabetes Mellitus é considerada fator predisponente para dermatomicoses, entretanto, não é bem descrito o porquê dessa associação, especialmente em se tratando de moradores em zona rural. Objetivo: Avaliar a ocorrência de dermatomicoses em diabéticos, maiores de 50 anos, residentes na zona rural de Ivatuba, Paraná, Brasil. Método: Participantes do programa HiperDia da Unidade Básica de Saúde responderam a um questionário e forneceram amostras de sangue e lesões tegumentares para investigação laboratorial. Resultados: Foram incluídos no estudo 45 indivíduos, dos quais 37 apresentavam lesões tegumentares e fungos foram detectados em 17 deles (45,9\%). Não houve diferença significativa nos parâmetros metabólicos avaliados, entre indivíduos com e sem micose, nem quanto a hábitos da vida rural. Enquanto isso, alguns fungos, aleatoriamente avaliados, foram resistentes à fagocitose in vitro e sobretudo à morte por fagócitos removidos de camundongos diabéticos. Conclusão: a taxa de dermatomicose encontrada em diabéticos foi alta e pode estar relacionada à falha na ativação de fagócitos.
\end{abstract}

Palavras-chave: Dermatomicoses; Diabetes Mellitus; Onicomicose.

\begin{abstract}
Diabetes mellitus is a predisposing factor for dermatomycosis, despite it is not yet completely understood, especially in regards to those living in rural areas. Objective: To evaluate the occurrence of dermatomycosis in diabetics, aged over 60 years old, living in the rural area of Ivatuba, Paraná, Brazil. Methods: Patients of HiperDia program answered a questionnaire and provided blood samples, and cutaneous lesions scraped, for laboratory investigation. Results: forty-five individuals were included in the study, of which 37 had cutaneous lesions and were investigated in the laboratory. Fungi were detected in 17 of them (45.9\%), with dermatophytes being the most frequent agents. There was no significant difference in the assessed metabolic parameters of individuals with and without mycosis, nor in terms of rural life habits, however some fungi sampled were resistant to phagocytosis and especially to death by phagocytes from diabetic animals. Conclusion: A high rate of dermatomycosis was detected in individuals with diabetes, probably related to the failure in the activation of phagocytes.
\end{abstract}

Keywords: Dermatomycoses; Diabetes Mellitus; Onychomycosis. 


\section{Resumen}

La diabetes mellitus se considera un factor predisponente para la dermatomicosis, sin embargo, el motivo de esta asociación no está bien descrito, especialmente cuando se trata de residentes rurales. Objetivo: Evaluar la ocurrencia de dermatomicosis en diabéticos, mayores de 50 años, residentes en el área rural de Ivatuba, Paraná, Brasil. Método: Los participantes del programa HiperDia de la Unidad Básica de Salud respondieron un cuestionario y entregaron muestras de sangre y tejidos blandos para la investigación de laboratorio. Resultados: Se incluyeron 45 individuos en el estudio, de los cuales 37 presentaban lesiones cutáneas y se detectaron hongos en 17 de ellos (45,9\%). No hubo diferencia significativa en los parámetros metabólicos evaluados, entre individuos con y sin micosis, ni en los hábitos de vida rural. Mientras tanto, algunos hongos, evaluados aleatoriamente, fueron resistentes a la fagocitosis in vitro y especialmente a la muerte por fagocitos extraídos de ratones diabéticos. Conclusión: la tasa de dermatomicosis encontrada en diabéticos fue alta y puede estar relacionada con falla en la activación de los fagocitos.

Palabras clave: Dermatomicosis; Diabetes Mellitus; Onicomicosis.

\section{Introdução}

Diabetes Mellitus (DM) é uma doença crônica, reconhecida como um sério problema de saúde pública mundial e representa ainda grande desafio para os sistemas de saúde. Em 2017, DM atingiu 425 milhões de pessoas no mundo todo, com projeção de aumentar para 510.8 milhões em 2030 (Basu et al., 2018) e para 693 milhões até 2045 (Cho et al., 2017). No Brasil, uma estimativa feita em 2013 sugeriu uma prevalência da doença de 6,2\%, aproximadamente 9 milhões de pessoas com diabetes no país (Iser et al., 2013). Além disso, todas as previsões apontam para aumento na prevalência de diabetes conforme a idade. Atualmente, para homens o pico de incidência de DM é entre 65-69 anos e mulheres entre 70 e 79 anos (Cho et al., 2017).

A maioria dos estudos sobre DM avalia pessoas vivendo em cidades, em ambiente rural, pouco é conhecido, um estudo que comparou idosos rurais e urbanos evidenciou que a prevalência de DM é maior nos idosos que residem na zona rural (da Silva et al., 2013). Além disso, uma revisão recente aponta para a necessidade de um olhar especial para o cuidado com essa doença em ambiente rural (da Silva et al., 2021). Porém, essa temática é ainda pouco explorada (Casagranda et al., 2018) e a lacuna é ainda maior se nesse cenário forem incluídas as dermatofitoses que, em tese, são favorecidas em ambiente rural e são importantes comorbidades da DM, entretanto, não foram encontrados estudos que envolvem triângulo de variáveis (DM, zona rural e micoses).

DM é uma doença grave associada com alta morbidade, tendo em vista as complicações e fatores de risco para sérios problemas associados (Wang et al., 2019). Esse cenário gera elevados custos para o serviço público e exige cuidados das equipes da atenção primária à saúde. Assim, é essencial o incentivo à publicações científicas na temática DM em moradores rurais, visando despertar nas equipes multiprofissionais a preocupação com o cuidado em saúde de forma holística, com o intuito de melhorar a adesão ao tratamento da própria DM e, também das comorbidades, incluindo as mais conhecidas como a Hipertensão Arterial Sistêmica (da Costa et al., 2021) e outras complicações ainda negligenciadas no mundo todo como as infecções fúngicas.

Entre as complicações crônicas do DM se destacam a doença renal crônica (Torres et al., 2017), o comprometimento cardiovascular (Busui et al., 2017), a retinopatia diabética (Xiao et al., 2017) e as neuropatias (Pai et al., 2018) as quais contribuem para elevados índices de mortalidade (Cho et al., 2017). Outra abordagem de alta relevância é a associação de DM com distúrbios cutâneos, conhecidos como dermopatias diabéticas tais como prurido, lesões bolhosas, necróticas, granulomatosas, vitiligo, líquens planos e outras (Lima et al., 2017). Nesse contexto, o risco aumentado de infecções bacterianas e fúngicas representa um dos fatores de grande preocupação. A síndrome do pé diabético é uma das complicações mais comuns nos pacientes com DM, representando duas a três vezes mais chances de morte (Mader et al., 2019). Essa síndrome é responsável pela queda significativa da qualidade de vida dos pacientes, além de elevado custo econômico, apesar disso, ainda é um problema negligenciado (Lavery et al., 2019). Recentemente foi comprovada a participação do pé diabético como porta de entrada para infecções fúngicas invasivas, com comprometimento de tecidos profundos (Öztürk et al., 2019), 
mas é evidente que existam outros fatores envolvidos tendo em vista a alta associação entre DM e candidemia (da Silva et al., 2013).

De fato, muitos autores têm considerado DM como fator de risco para infecções fúngicas, envolvendo desde micoses superficiais até infecções sistêmicas graves e fatais (Foss et al., 2005; Poradzka et al., 2013; Dahlke et al., 2015; Akkus et al., 2016; Eba et al., 2016; Oz et al., 2017; Gupta et al., 2017; Salazar-Tamayo et al., 2017; Sultana et al., 2018; Lavery et al., 2019). Essas infecções são classificadas como oportunistas e são mais associadas ao pobre controle do DM, a taxas elevadas de glicemia e são frequentes como manifestações clínicas da pele e unhas (Foss et al., 2005; Poradzka et al., 2013; Dahlke et al., 2015; Akkus et al., 2016; Assadamongkol et al., 2016).

Apesar disso, são escassos os estudos que comprovem documentalmente a associação entre DM e dermatomicoses, pois essa relação, especialmente em população rural, ainda é praticamente desconhecida. Assim, o objetivo deste estudo foi avaliar a ocorrência de dermatomicoses em indivíduos diabéticos, com mais de 50 anos de idade, residentes na zona rural do município de Ivatuba - Paraná, Brasil; bem como caracterizar laboratorialmente estes pacientes quanto ao perfil glicêmico, lipídico e função renal, além de desenvolver estudos in vitro com alguns dos fungos isolados, frente a fagócitos de hospedeiro diabético. Este estudo, em conjunto, visou melhor entender os mecanismos que possam explicar a associação entre DM e dermatomicoses.

\section{Metodologia}

\section{Abordagem inicial e dados descritivos}

Caracterização do estudo - foi realizado estudo transversal, a partir do cadastro de pacientes diabéticos da Unidade Básica de Saúde (UBS) do serviço público de Ivatuba. Este é um município de pequeno porte, localizado na região Noroeste do Estado do Paraná, Brasil. Segundo o senso de 2019 tem pouco mais de 3 mil habitantes dos quais, aproximadamente 25\% residem na zona rural. O serviço de saúde pública dispõe de uma UBS com Equipes da Estratégia Saúde da Família (EqSF) que atendem toda a população. Este estudo foi realizado por pesquisadores do Programa de Pós-Graduação em Biociências e Fisiopatologia da UEM em parceria com a Secretaria de Saúde Pública de Ivatuba, e aprovado pelo Comitê Permanente de Ética em Pesquisa com Seres Humanos (COPEP/UEM), parecer número 2.754.746.

População de estudo - inicialmente foram abordados todos os pacientes inscritos como diabéticos, os quais são assistidos pelo programa HiperDia do Ministério da Saúde do Brasil. A abordagem inicial, a apresentação do projeto e o convite aos pacientes foram feitos durante um dos encontros regulares deste programa, coordenados pela UBS. Com a assistência da EqSF, foram considerados como residentes na zona rural aqueles indivíduos fixados em regiões definidas pelo município como "rural", cuja renda (sistema produtivo ou aposentadoria) é em função de atividades agrícolas e as residências eram no estilo de "sítios" distantes uma das outras em pelo menos 500 metros em média, com densidade demográfica média inferior a 15 habitantes por quilômetro quadrado $\left(\mathrm{hab} / \mathrm{km}^{2}\right)$.

Do total de pacientes inscritos, à época no programa HiperDia $(n=139)$ foram excluídos: os com idade inferior a 50 anos, os residentes na região urbana do município, os que faleceram ou mudaram de residência durante o período de avaliação, os acamados, os ausentes por qualquer razão e os que não deram consentimento para o estudo. 
Figura 1. Distribuição dos pacientes assistidos na UBS, pelo programa HiperDia: demonstrando em A: os incluídos no estudo e representação dos pacientes participantes do projeto, número e porcentagem de pacientes com dermatomicoses. ** Fungos isolados (Candida sp) de uma paciente com onicomicose em unha da mão, com paroníquia crônica, foram empregados para estudos de resistência à fagocitose e Killing por fagócitos obtidos de camundongos diabéticos. Em B são detalhados os critérios para exclusão de pacientes no estudo.
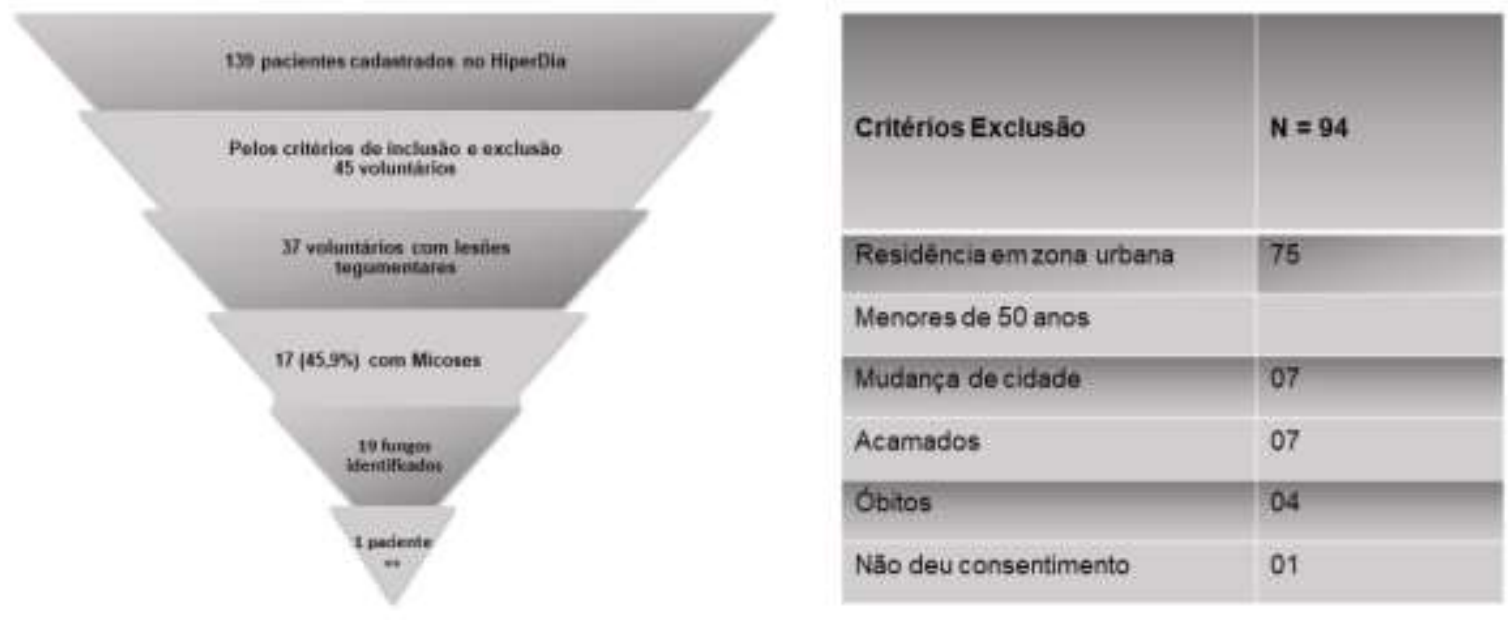

Fonte: Autores.

Como é mostrado na Figura 1, foram incluídos 45 voluntários, os quais foram abordados em visitas domiciliares, pelos pesquisadores no período entre setembro de 2018 e fevereiro 2019, para exposição detalhada do estudo. Na ocasião foi obtido o Termo de Consentimento Livre e Esclarecido e colhida a assinatura dos indivíduos com a oficialização da adesão ao estudo. Esses voluntários responderam a um questionário sobre questões relacionadas à presença de lesões tegumentares e fatores de risco para dermatomicoses. As informações sobre lesões tegumentares foram autorreferidas, sem exigência de comprovação ou diagnóstico clínico. Dermatomicoses, para esse estudo, foram consideradas todas as lesões superficiais e cutâneas localizadas na pele ou anexos, das quais posteriormente foram isolados fungos de interesse médico.

\section{Estudo experimental}

Processamento das amostras biológicas - os 37 pacientes que manifestavam lesões tegumentares forneceram amostras biológicas de sangue e escamas de pele ou unhas para confirmação laboratorial (bioquímica e micológica respectivamente). Amostras de sangue venoso total, coletadas em jejum, foram processadas com as seguintes intenções: avaliar as condições do DM: dosagem de glicose plasmática de jejum e hemoglobina glicada (Gross et al., 2002). Avaliação do perfil lipídico: dosagem sérica de colesterol total e frações (HDL, LDL e VLDL). Avaliação da função renal: dosagem de creatinina e ureia, todas essas determinações foram feitas por método espectrofotômetro semianalítico BIOPLUS, utilizando método de identificação enzimático e colorimétrico.

Escamas de pele (cabeça, face, membros superiores e inferiores ou unhas) foram obtidas por meio de raspagem, após antissepsia com álcool 70\%. Essas amostras foram usadas na investigação quanto à presença de fungos, visando a caracterização laboratorial de possíveis dermatomicoses. Foi realizado o exame micológico direto (EMD), que é a busca de evidências de estruturas fúngicas na amostra biológica. Deste material também foi feita a cultura para fungos com identificação dos mesmos. Para o EMD cada amostra foi colocada entre lâmina e lamínula, com KOH 20\% adicionado de azul de Evans 0,5\% e mantidas em câmara úmida por uma noite, para clarificação. As culturas para fungos foram feitas em tubos contendo Sabouraud Dextrose Ágar (SDA), KASVI, Liofilchem, Itália acrescido do antibiótico cloranfenicol e também em Ágar seletivo 
para dermatófitos Ágar Mycosel, BBL Le pont de Claix, França. Foram utilizados três tubos para cada um dos meios de cultivo onde foram feitas três inoculações das escamas de cada sítio, em pontos equidistantes, totalizando 18 inoculações para cada amostra. Todos esses tubos foram incubados em estufa, a $25^{\circ} \mathrm{C}$, por até 30 dias. Essas culturas foram monitoradas em dias alternados (para determinar exatamente o tempo que cada fungo necessita para crescer) e, imediatamente, foi iniciado o processo de identificação dos fungos. O gênero e espécie de cada colônia fúngica desenvolvida foram determinados por meio dos métodos clássicos (macro e micromorfologia) segundo Larone, 2011 e confirmação por MALDI TOFF (Normand et al., 2017). Culturas que não desenvolveram fungos em 30 dias foram descartadas e consideradas negativas.

Dois isolados fúngicos identificados como leveduras do gênero Candida, os quais foram isolados de uma paciente feminina que apresentava onicomicose em unha da mão, com paroníquia crônica, comum em DM, foram usados para estudos sobre a interação frente a fagócitos isolados de camundongos diabéticos.

Estudos in vitro de fagocitose e Killing - Fagócitos peritoneais de camundongos diabéticos (De Zuani et al., 2018) foram usados em experimentos visando avaliar a resistência de alguns fungos a esses fagócitos, baseados em Diez-Orejas et al. (2018), com algumas modificações. Brevemente, suspensões de C. albicans e C. parapsilosis foram preparadas e ajustadas em PBS para conter $2 \times 10^{4}$ unidades formadoras de colônias por mililitro (UFC/mL). Co-culturas da suspensão de cada fungo e dos fagócitos na proporção 5:1 foram feitas em microplacas esterilizadas KASVI, China) contendo lamínulas redondas e mantidos por $2 \mathrm{~h}$ na estufa de $\mathrm{CO}_{2}$. O índice de fagocitose foi determinado por microscopia após coloração do material aderido nas lamínulas pelo corante hematológico rápido Panótico (Instant-Prov, Newprov, Piraquara, Brazil). Foram avaliados 200 fagócitos e determinada a percentagem de fagocitose (ou seja, de células que tinham leveduras interiorizadas). Para o ensaio de Killing, uma suspensão preparada como descrito anteriormente foi opsonizada com soro fetal bovino estéril (Sigma Aldrich, EUA), na proporção de $200 \mu \mathrm{L}$ de soro para $5 \mathrm{~mL}$ da suspensão de fungos. A relação fagócito/fungo foi 1:10 sendo C. albicans $\left(2 \times 10^{6}\right)$ e $C$. parapsilosis $\left(2 \times 10^{7}\right) \mathrm{UFC} / \mathrm{mL}$. As culturas foram incubadas em shaker a $37^{\circ} \mathrm{C}$, sob agitação (100 rpm) por 02 horas. Em tempos determinados (0, 60 e 120) minutos, foram coletados $100 \mu \mathrm{L}$ de cada co- cultura, os quais foram diluídos em água destilada gelada para lise dos fagócitos e liberação dos micro-organismos internalizados. O material resultante foi plaqueado em SDA. Após incubação a $35^{\circ} \mathrm{C}$ por $24-48 \mathrm{~h}$, foi determinado o número de $\mathrm{UFC} / \mathrm{mL}$ e os resultados foram expressos em porcentagem de sobrevivência. Fagócitos peritoneais extraídos de animais de mesma idade, peso e raça, porém não diabéticos foram usados para comparação.

Análise estatística - para as análises dos ensaios de Killing e fagocitose foi utilizado o teste ANOVA seguido da múltipla comparação pelo teste de Bonferroni. A análise de correlação apresentada na Tabela 1 foi realizada utilizando o teste Chi-square ( $\chi 2$ ) com o cálculo de Odds Ratio (OR) e intervalo de confiança de $95 \%$. Valores de $\mathrm{p}<0.05$ foram considerados estatisticamente significativos. Para as análises foi utilizado o software Graphpad Prism 7. Os resultados dos ensaios in vitro foram obtidos em três ensaios independentes, feitos em triplicata.

\section{Resultados}

Na UBS de Ivatuba estavam cadastrados 139 pacientes com diabetes; esse total foi considerado no início do estudo. Mas atendendo os critérios de inclusão e exclusão, 45 deles (33,3\%) participaram do estudo. Entre os 45 participantes, todos tiveram o diagnóstico de DM há mais de cinco anos, oito eram insulinos dependentes e nenhum deles tinha "pé diabético". Entretanto, a maior parte, $37(82,20 \%)$ apresentavam lesões tegumentares e este grupo constituiu o objeto deste estudo experimental.

As características gerais da população de estudo estão resumidas na Tabela 1. A média de idade dos 37 indivíduos foi de 66,6 anos, sem diferença estatística quanto à presença de dermatomicoses $(\mathrm{p}=0,889)$. Houve predomínio de mulheres $(81,08 \%) \mathrm{n}=30$ em 13 dessas, a dermatomicose foi confirmada laboratorialmente $(43,33 \%)$. Em relação aos homens, eram 
minoria, apenas $(18,91 \%) \mathrm{n}=7$, sendo que quatro deles $(57,14 \%)$ tinham dermatomicose, contudo, esses dados não conferem diferença significativa entre os gêneros $(p=0,222)$.

Tabela 1 - Informações antropométricas e alguns hábitos dos 37 pacientes com diabetes que tinham lesões tegumentares, conforme a presença ou ausência de dermatomicose.

\begin{tabular}{lcccc}
\hline Características & Total* $^{*}$ & Dermatomicose** & Sem micose & P \\
\hline Geral & 37 & 17 & 20 & \\
Homens & 7 & 4 & 3 & \\
Mulheres & 30 & 13 & 17 & \\
Idade (anos) & $66,6( \pm 9,6)$ & $64,1( \pm 9,5)$ & $68,7( \pm 9,2)$ & 0,889 \\
Contato com terra/solo & 32 & 13 & 19 & ------------ \\
Contato com Animais & 26 & 13 & 13 & \\
& & & & \\
Contato com rios/lagos & 8 & 4 & 4 & \\
\hline
\end{tabular}

* Número total ou média (DP \pm ); **Fungos detectados: pelo Exame Micológico Direto e/ou cultura para fungos. Fonte: Autores.

A frequência de dermatomicose em diabéticos foi de 45,9\%, pois fungos foram detectados em 17 dos 37 indivíduos. Entre esses 17 pacientes, cinco eram insulinos dependentes, um fazia o controle do DM sem medicação e os demais faziam uso de hipoglicemiantes orais. Apesar dessa população residir em zona rural, os hábitos comuns que constam na Tabela 1 (como andar descalço e contato com o solo, água e animais domésticos) não foram contundentes e não estão relacionados à presença de micoses.

Conforme mostra a Tabela 2, o valor médio da glicemia em jejum, dos 37 pacientes, foi 147,1 mg/dL e, no grupo com micose comprovada, esse valor foi maior do que naqueles em que fungos não foram detectados $168,9 \mathrm{mg} / \mathrm{dL}$ e $128,5 \mathrm{mg} / \mathrm{dL}$ respectivamente $(\mathrm{p}=0,050)$. Já, em relação ao controle da DM, os resultados de HG sugerem a manutenção crônica da glicose sérica em níveis elevados $(7,2)$ sem diferença significativa para a presença ou não de dermatomicose $(p=0,079)$. 
Tabela 2 - Parâmetros bioquímicas dos 37 pacientes com diabetes que tinham lesões tegumentares, conforme a presença ou ausência de dermatomicose.

\begin{tabular}{lcccc}
\hline Características & Total* & Dermatomicose $\mathbf{F}^{* *}$ & Sem micose & P \\
\hline Glicemia em jejum & $147,1( \pm 58,2)$ & $168,9( \pm 66,7)$ & $128,5( \pm 41,7)$ & 0,050 \\
Hemoglobina Glicada & $7,2( \pm 1,5)$ & $7,3( \pm 1,1)$ & $7,1( \pm 1,7)$ & 0,079 \\
Colesterol total & $141,9( \pm 52,4)$ & $136,0( \pm 55,6)$ & $147,0( \pm 49,1)$ & 0,584 \\
Triglicérides & $189,4( \pm 108,1)$ & $201,1( \pm 91,2)$ & $179,6( \pm 119,8)$ & 0,283 \\
HDL & $45,3( \pm 16,0)$ & $47,2( \pm 17,0)$ & $43,8( \pm 15,0)$ & 0,585 \\
LDL & $66,1( \pm 45,1)$ & $52,6( \pm 48,1)$ & $77,6( \pm 38,9)$ & 0,366 \\
VLDL & $36,8( \pm 21,3)$ & $37,9( \pm 17,7)$ & $35,9( \pm 24,0)$ & 0,235 \\
Ureia & $45( \pm 17,3)$ & $42,5( \pm 14,6)$ & $47,1( \pm 19,1)$ & 0,297 \\
Creatinina & $1,0( \pm 0,3)$ & $0,9( \pm 0,2)$ & $1,1( \pm 0,4)$ & 0,026 \\
\hline
\end{tabular}

* Valor médio (DP \pm ); **Fungos detectados: pelo Exame Micológico Direto e/ou cultura para fungos; Fonte: Autores.

Também não foi detectada diferença significativa entre os parâmetros bioquímicos relacionados ao perfil lipídico. Quanto à função renal, apesar de os pacientes sem micose apresentarem um valor estatisticamente maior para creatinina $(\mathrm{p}=$ $0,026)$, os valores absolutos, bem como os da ureia estavam dentro do limite da normalidade.

A confirmação laboratorial de dermatomicose ocorreu através de duas formas (EMD e cultura); inicialmente foi investigada a presença de fungos diretamente no material biológico, o qual revelou estruturas compatíveis. O desenvolvimento dos fungos em cultura e identificação em nível de gênero e espécie permitiu confirmar dermatomicose em 17 indivíduos, sendo que em dois deles foram encontradas duas espécies, totalizando 19 isolados clínicos cuja distribuição está mostrada na Tabela 3. Dermatófitos foi o grupo de fungos predominante, seguido pelas leveduras, enquanto fungos filamentosos não dermatófitos (FFND) foram isolados em menor frequência. Trichophyton rubrum, Candida albicans e Fusarium oxysporum foram as espécies mais frequentes em cada grupo de fungos respectivamente.

O perfil dos 17 pacientes com diabetes e com dermatomicose é apresentado na Tabela 4, ou seja, a idade variou entre 52 e 95 anos com média de 64,1. O índice de HG médio foi 7,3 variando de 4,9 a 9,3. Ao todo foram detectados fungos em 18 sítios anatômicos, sendo as unhas mais frequentemente envolvidas (dos pés $=13$ e das mãos $=2$ ). Embora vários pacientes tivessem lesões tegumentares em mais de um sítio, os quais foram investigados separadamente quanto à presença de fungos, somente em um paciente foram encontrados fungos em dois sítios diferentes.

A presença de dermatófitos ocorreu independente do gênero do paciente, pois foram detectados em todos os quatro homens que tinham micose, também, em seis das 13 mulheres. Já os fungos oportunistas, incluindo as leveduras $(C$. parapsilosis, C. albicans e Malassezia spp.) bem como Fusarium spp. foram encontrados exclusivamente em pessoas do gênero feminino. 
Tabela 3 - Distribuição dos fungos isolados de dermatomicoses em pacientes diabéticos residentes na zona rural de Ivatuba, PR, Brasil.

\begin{tabular}{cll}
\hline & Fungos detectados & $\mathbf{N}^{\mathbf{0}} \mathbf{d e}$ isolados \\
\hline $\begin{array}{c}\text { Dermatófitos } \\
53 \%\end{array}$ & Trichophyton rubrum & 8 \\
& Trichophyton mentagrophytes & 2 \\
Leveduras & Candida albicans & 3 \\
$31 \%$ & Candida parapsilosis & 1 \\
& Malassezia spp & 2 \\
FFND & Fusarium solani & 1 \\
\hline TOTAL & Fusarium oxysporum & 2 \\
\hline
\end{tabular}

Fonte: Autores.

A tabela 5 mostra os medicamentos que a população de estudo fazia uso na época da coleta de dados. Em relação aos antifúngicos, na época da coleta de dados, apenas um dos pacientes com micose confirmada (paciente número 2) estava fazendo uso de Icaden, mas $(35,30 \%) \mathrm{n}=6$ relataram ter usado antifúngicos no passado, esses dados estão especificados para cada paciente na Tabela 4.

Detalhadamente, quatro indivíduos $(1,8,14$ e 17) declararam já ter feito tratamento antifúngico, todos com prescrição médica. Enquanto dois deles ( 9 e 15) declararam ter usado formulações caseiras como (solução de álcool com cloro, Lysoform, óleo de cravo etc.) visando o tratamento das respectivas lesões. Entre os 20 pacientes dos quais não foi detectada dermatomicose, seis relataram terem usado no passado alguma medicação antifúngica prescrita por médico (Canesten, Cetoconazol, Terbinafina tópica) ou por automedicação (Ciclopirox olamina, própolis). 
Tabela 4 - Distribuição dos 17 pacientes diabéticos com dermatomicoses.

\begin{tabular}{|c|c|c|c|c|c|c|c|c|}
\hline $\mathbf{A}$ & B & C & Insulina & $\begin{array}{l}\text { HG } \\
(\%)\end{array}$ & Sítio & EMD & Cultura & $\begin{array}{l}\text { Antifúngico } \\
\text { prévio }\end{array}$ \\
\hline 1 & M & 61 & Não & 7,4 & unhas do pé & $\mathrm{FM}$ & $\begin{array}{l}\text { Trichophyton } \\
\text { rubrum }\end{array}$ & $\begin{array}{l}\text { Fluconazol } \\
\text { creme }\end{array}$ \\
\hline 2 & $\mathrm{~F}$ & 63 & Não & 6,9 & unha do pé & FM & $\begin{array}{l}\text { Fusarium } \\
\text { oxysporum }\end{array}$ & $\begin{array}{l}\text { Icaden } \\
\text { creme* }\end{array}$ \\
\hline 3 & $\mathrm{~F}$ & 72 & Não & 4,9 & unha do pé & $\mathrm{EF}$ & $\begin{array}{l}\text { Trichophyton } \\
\text { rubrum }\end{array}$ & Não \\
\hline 4 & $\mathrm{~F}$ & 59 & Sim & 9,3 & pele (interdigital) & $\mathrm{EF}$ & Candida albicans & Não \\
\hline 5 & M & 55 & Não & 6,7 & unha do pé & FM & $\begin{array}{l}\text { Trichophyton } \\
\text { rubrum }\end{array}$ & Não \\
\hline 6 & $\mathrm{~F}$ & 62 & Não & 5,6 & unha do pé & $\mathrm{EF}$ & Fusarium solani & Não \\
\hline 7 & $\mathrm{~F}$ & 52 & Não & 7,1 & pele & LEV & Malassezia sp & Não \\
\hline 8 & $\mathrm{~F}$ & 64 & Não & 7,0 & unha do pé & FM & $\begin{array}{l}\text { Trichophyton } \\
\text { mentagrophytes }\end{array}$ & $\begin{array}{l}\text { Terbinafina } \\
\text { creme }\end{array}$ \\
\hline 9 & $\mathrm{~F}$ & 53 & Não & 8,8 & unha do pé & FM & $\begin{array}{l}\text { Trichophyton } \\
\text { rubrum }\end{array}$ & $\begin{array}{l}\text { Formulação } \\
\text { caseira }\end{array}$ \\
\hline 10 & $\mathrm{~F}$ & 66 & Sim & 7,8 & unha da mão & LEV & $\begin{array}{l}\text { Candida albicans e } \\
\text { Candida } \\
\text { parapsilosis }\end{array}$ & Não \\
\hline 11 & $\mathrm{~F}$ & 68 & Não & 7,2 & unha do pé & FM & $\begin{array}{l}\text { Trichophyton } \\
\text { rubrum }\end{array}$ & Não \\
\hline 12 & M & 70 & Não & 8,9 & unha do pé & $\mathrm{EF}$ & $\begin{array}{l}\text { Trichophyton } \\
\text { mentagrophytes }\end{array}$ & Não \\
\hline 13 & $\mathrm{~F}$ & 57 & Não & 7,4 & unha do pé & $\mathrm{EF}$ & $\begin{array}{l}\text { Fusarium } \\
\text { oxysporum }\end{array}$ & Não \\
\hline 14 & $\mathrm{~F}$ & 66 & Sim & 7,3 & unha do pé & FM & $\begin{array}{l}\text { Trichophyton } \\
\text { rubrum }\end{array}$ & $\begin{array}{l}\text { Ciclopirox } \\
\text { olamina }\end{array}$ \\
\hline 15 & $\mathrm{~F}$ & 64 & Sim & 8,1 & unha da mão & LEV & Candida albicans & $\begin{array}{l}\text { Formulação } \\
\text { caseira }\end{array}$ \\
\hline 16 & $\mathrm{~F}$ & 95 & Não & 6,8 & $\begin{array}{l}\text { unha do pé } \\
\text { couro cabeludo }\end{array}$ & $\begin{array}{l}\text { FM } \\
\text { LEV }\end{array}$ & $\begin{array}{l}\text { Trichophyton } \\
\text { rubrum e } \\
\text { Malassezia sp }\end{array}$ & Não \\
\hline 17 & M & 64 & Sim & 7,0 & unha do pé & FM & $\begin{array}{l}\text { Trichophyton } \\
\text { rubrum }\end{array}$ & Clotrimix \\
\hline
\end{tabular}

$\mathrm{A}=$ Paciente; $\mathrm{B}=\mathrm{SEXO}$ (Masculino e Feminino); $\mathrm{C}=\mathrm{IDADE}$ (anos); $\mathrm{FM}=$ filamentos micelianos; LEV = leveduras; HG= Hemogloblina glicada; $\mathrm{FNV}=$ fungos não visualizados; $\mathrm{AEF}=$ Abundantes estruturas fúngicas. $*$ único em tratamento no dia da coleta de dados. Fonte: Autores. 
Tabela 5 - Distribuição dos medicamentos relatados pelos 37 participantes da pesquisa

\begin{tabular}{|c|c|c|c|}
\hline Agravo & Medicamentos & $\begin{array}{c}\text { Dermatomicose } \\
(\%)\end{array}$ & $\begin{array}{c}\text { Sem micose } \\
(\%)\end{array}$ \\
\hline \multirow{7}{*}{ Diabetes Mellitus } & Insulina & 29 & 15 \\
\hline & Metformina & 35 & 70 \\
\hline & Azukon & 6 & 5 \\
\hline & Nesina & 6 & 0 \\
\hline & Losartana & 41 & 55 \\
\hline & Benicar & 6 & 0 \\
\hline & Propanolol & 6 & 5 \\
\hline \multirow[t]{5}{*}{ Pressão arterial } & Hidroclorotiazida & 18 & 5 \\
\hline & Furosemida & 6 & 5 \\
\hline & Antibióticos & 12 & 15 \\
\hline & Estatinas & 18 & 5 \\
\hline & Hormônios tireóidianos & 18 & 5 \\
\hline \multirow[t]{4}{*}{ Outros } & Somalgin/Diazepan & 18 & 5 \\
\hline & Paracetamol & 6 & 10 \\
\hline & Ecasil & 6 & 10 \\
\hline & Protetor gástrico & 6 & 15 \\
\hline
\end{tabular}

Fonte: Autores.

Os resultados da avaliação da relação de fungo frente a células fagocíticas de hospedeiro diabético são apresentados na Fig 2. C. albicans, isolada de paciente diabético, foi reconhecida pelos fagócitos da mesma forma que a cepa referência ATCC. Os fagócitos foram capazes de internalizar ambos os isolados, porém, falharam nos eventos microbicidas (Fig 2B), pois não houve diferença significativa entre leveduras viáveis expostas ou não aos fagócitos. Em relação a $C$. parapsilosis, apesar de menor taxa de internalização (Fig 2C), também foi observado resistência à morte. Fagócitos de animais não diabéticos, usados como controle, foram mais eficientes em inativar essas leveduras, tanto os isolados clínicos quanto as de referência (dados não mostrados). 
Figura 2 - Resistência de leveduras isoladas de paciente diabético ( $\mathrm{Ca} \mathrm{W}=$ Candida albicans e $\mathrm{Cp} \mathrm{W}=$ Candida parapsilosis) à fagocitose (A e C) e morte (B e D) quando expostas in vitro à ação de fagócitos peritoneais de camundongos Balb/C diabéticos, em comparação com as respectivas cepas de referência ATCC. Diferença estatística em nível de significância 0,05 detectada apenas em relação à fagocitose por $C$. parapsilosis isolada de paciente diabético.

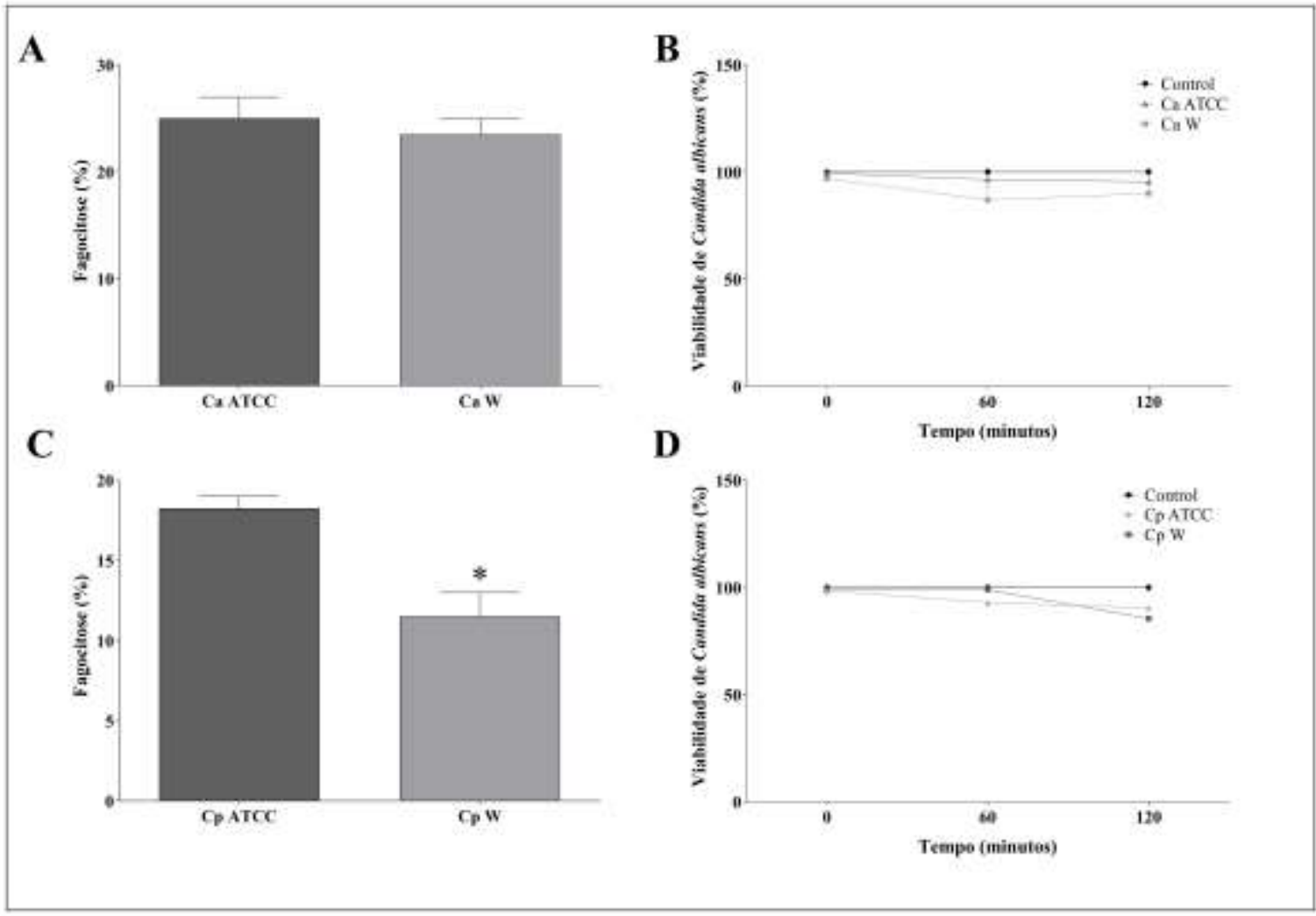

Fonte: Autores.

\section{Discussão}

A vida moderna, com fácil acesso das pessoas aos serviços de saúde, tem contribuído para o aumento da expectativa de vida da população. Entretanto, prolongar a vida torna os indivíduos mais vulneráveis a doenças crônicas, entre outras o DM e suas comorbidades. O presente estudo focou em DM e dermatomicoses, por tratar-se de uma associação admitida, porém pouco documentada. A prevalência dessas micoses é considerada maior entre diabéticos (Foss et al., 2005; Sanches et al., 2019), apesar disso, essa relação ainda é pouco avaliada, especialmente em comunidades rurais.

No presente estudo a frequência de dermatomicose foi de 45,9\%; não encontramos outros estudos com design semelhante, realizados em populações rurais para comparação. Mas no geral, estudos publicados indicam que esse valor está na mesma faixa encontrada em uma revisão sistemática (Sanches et al., 2019). Além disso, é equivalente aos 46\% encontrados, em outro estudo no sul do Brasil (Dahlke et al., 2015) e é superior aos 31,9\% encontrados na Tailândia (Assadamongkol et al., 2016). Essa reflexão nos permite inferir que a prevalência de dermatomicoses em diabéticos independe da localização da moradia (rural ou urbana).

Por outro lado, esta mesma comparação, torna nossos achados mais relevantes, uma vez que os pacientes incluídos no presente estudo não passaram por triagem clínica quanto à suspeita de micose, todos os pacientes cadastrados foram convidados a participar da pesquisa e as dermatomicoses foram autorreferidas também, o aceite ou não a participar do estudo, foi espontâneo. Ou seja, todas essas dermatomicoses não tinham sido suspeitas ou consideradas no atendimento de rotina. É 
inegável, entretanto, a possibilidade da frequência encontrada estar superestimada tendo em vista que indivíduos acometidos por lesões tegumentares podem ter aderido mais facilmente ao nosso convite do que os que não tinham essa queixa.

Em nossa casuística, a idade média, incluindo homens e mulheres, ficou próxima a 65 anos, em concordância com os achados em diabéticos de população rural do sul do Brasil (Casagranda et al., 2018). Houve predomínio de mulheres, também concordando com outros autores (Iser et al., 2013; Assadamongkol et al., 2016; Casagranda et al., 2018) o que pode ser atribuído à maior procura das mulheres pelos serviços de saúde. Porém, isso não é regra, pois outros autores têm encontrado DM com mais frequência no gênero masculino (Akkus et al., 2016; Oz et al., 2017).

Conforme a Tabela 2, diabéticos com dermatomicoses tinham níveis glicêmicos mais elevados do que aqueles em que não foi detectado fungos, com resultado no limite da significância $(\mathrm{p}=0,050)$ e sem diferença significativa quanto ao controle glicêmico $(p=0,079)$. Interessantemente, de oito indivíduos insulinos dependentes do grupo todo, verificamos que cinco deles (62,5\%) tinham dermatomicose (Tabela 4). Além disso, entre os indivíduos insulinos dependentes e portadores de micose, o valor da HG foi ligeiramente maior (7,9\%) em comparação aos demais (7,1\%). Esse achado está de acordo com outros autores (Foss et al., 2005; Akkus et al., 2016; Salazar-Tamayo et al., 2017), os quais assumem que micoses estariam associadas ao pobre controle da DM.

Quanto ao perfil lipídico, os resultados estão dentro da normalidade, portanto, sem destaque em relação à presença ou não de micose. Os dados bioquímicos encontrados sugerem uma população metabolicamente controlada, possivelmente relacionada aos hábitos de vida saudáveis da zona rural. Além disso, é preciso destacar que todo o grupo de pacientes faz parte do programa HiperDia do Ministério da Saúde, onde são acompanhados rotineiramente pelo serviço de saúde, garantindo maior controle da DM e das condições fisiológicas e, consequentemente, das comorbidades. Corroborando essa hipótese destacamos a ausência de úlceras ou pé diabético na população de estudo. A ausência de comorbidades também pode ser inferida levando em conta os medicamentos usados pelo grupo (Tabela 5). Aparentemente, os medicamentos de uso contínuo não indicam nenhum outro tipo de imunocomprometimento, além do DM.

Entre as dermatomicoses confirmadas em nosso estudo (Tabela 4), as mais frequentes foram as onicomicoses (OM). A frequência de OM encontrada entre os 45 indivíduos com DM foi 33,33\% e, considerando os 37 voluntários que manifestavam lesões tegumentares essa porcentagem sobe para 40,54\% bem superiores à prevalência estimada para a população geral ao redor do mundo que é 5,5\% (Lipner et al., 2019) porém, inferior ao encontrado em diabéticos de Camarões que foi 50.7\% (Eba et al., 2016). Nossos dados estão de acordo com a maioria dos autores, pois OM realmente é a dermatomicose mais frequente na população geral e aumenta com a idade (Eba et al., 2017) em diabéticos ou não (Oz et al., 2017; Sultana et al., 2018). Entre as unhas, a dos pés foram mais acometidas, achado também em consonância com o observado de modo geral na literatura.

Chamou atenção o descaso com que as dermatofitoses vêm sendo abordadas na prática desses pacientes, pois nenhum dos 17 indivíduos descritos na Tabela 4 tinha diagnóstico laboratorial de micose. Apenas um teve suspeita clínica e estava em tratamento, quatro declararam ter usado antifúngicos no passado com prescrição médica, não foram capazes de precisar há quanto tempo, mas relataram que não obtiveram melhora com tais tratamentos. Esse dado é preocupante, considerando DM como fator de risco para infecções fúngicas (Gupta et al., 2017; Salazar-Tamayo et al., 2017). Além disso, está bem descrito o risco de alguns fungos, como as leveduras, evoluírem para infecções invasivas graves e fatais (da Silva et al., 2013). Bem como está confirmado que Fusarium spp podem usar a unha infectada como porta de entrada para disseminação sistêmica (Varon et al., 2014).

Entre os agentes isolados, o grupo dos dermatófitos foi predominante, contrastando com os dados encontrados em Blumenau (Dahlke et al., 2015). Naquele estudo, os fungos predominantemente encontrados em diabéticos foram leveduras do gênero Candida spp (86\%) e apenas 14\% eram dermatófitos. Essa discrepância pode ser explicada pelo fato de Blumenau, 
apesar de também ser localizada no sul do Brasil, ter uma variação sazonal importante em relação à umidade, que parece favorecer o desenvolvimento de leveduras. A espécie Candida albicans foi a mais comum, no grupo das leveduras, nos dois estudos.

Trichophyton rubrum foi o agente mais isolado no presente estudo, corroborando também os achados mais frequentes no mundo todo (Nenoff et al., 2014; Eba et al., 2016; Oz et al., 2017; Sultana et al., 2018; Babayani et al., 2018; Lipner et al., 2019). Interessantemente este é um fungo antropofílico, bastante adaptado com a vida urbana, associado ao uso de calçados fechados e manutenção em domicílios urbanos (ambientes geralmente mais interiorizados, com carpetes, tapetes etc...). Enquanto para a zona rural, seria esperado encontrar fungos zoofílicos, os quais não foram isolados neste estudo. Assim, aparentemente não há diferenças relevantes quanto à presença de dermatomicoses em pacientes diabéticos residentes em zona rural ou urbana, o que pode estar atribuído, pelo menos em parte, à modernidade, ao tipo de moradia e hábitos cotidianos, que parecem expor igualmente ao risco de contaminação por fungos. Entretanto, é de nota o bom nível quanto à qualidade de vida desses indivíduos, inferida indiretamente pelos dados levantados como parâmetros bioquímicos, medicamentos utilizados, ausência de lesões complicadas, entre outros.

Fusarium spp foi o único FFND encontrado, esse gênero é associado com atividades agrícolas e, tem sido isolado com frequência na região Noroeste do Paraná, também com destaque para a espécie F. oxysporum (Galletti et al., 2015). É capaz de invadir a unha usando-a como única fonte nutricional (Veiga et al., 2018) e, também foi encontrado em pacientes diabéticos na Tailândia (Assadamongkol et al. 2016).

Apesar da alta frequência de dermatomicoses entre os diabéticos avaliados não encontramos nenhum fator que justificasse sua associação com DM. Então, optamos por fazer um estudo laboratorial complementar visando avaliar o comportamento de alguns dos fungos isolados, quanto à resistência à fagocitose e Killing por fagócitos peritoneais de camundongos diabéticos. Apesar de os dermatófitos terem sido os fungos mais frequentes, nossa escolha foi por leveduras pois, o gênero Candida diferentemente dos dermatófitos, pode evoluir a partir de distúrbios cutâneos para causar infecções graves e fatais como a candidemia (da Silva et al., 2013). Além disso, o quadro clínico da paciente escolhida era tipicamente associado a DM: lesões em unhas de mãos, com paroníquia crônica, em mulher diabética, com 66 anos de idade, dona de casa (Dorko et al., 2005; Dahlke et al., 2015; Syzon et al., 2019; Erraji et al., 2020) e ela não tinha recebido tratamento antifúngico. Ambos os isolados clínicos C. albicans e C. parapsilosis ( $\mathrm{CaW}$ e $\mathrm{CpW}$ ) foram mais sensíveis à fagocitose do que as respectivas cepas de referência, principalmente $C$. parapsilosis cuja taxa de fagocitose foi 1,5 vezes menor comparado à cepa ATCC.

Considerando que a atividade microbicida é o evento sequencial à fagocitose, foi avaliado o desfecho dessa interação determinando a taxa de morte (Killing). Nesse sentido, as Figuras 2 B e D mostram que essas células fagocíticas foram deficientes na atividade microbicida sobre os dois isolados clínicos, independente da taxa de fagocitose. C. parapsilosis foi mais eficiente em evadir dos fagócitos de animais diabéticos do que C. albicans (Fig. 2C). De fato, a literatura mostra que a atividade microbicida dos fagócitos de pacientes com DM é menos eficiente devido às alterações metabólicas decorrentes da presença da hiperglicemia crônica (Assadamongkol et al., 2016). Adicionalmente, as altas taxas de HG parecem contribuir para a permanência fúngica nesses hospedeiros e aumento das infecções cutâneas, as quais teriam o risco potencial de evoluírem para quadros sistêmicos invasivos e graves.

\section{Conclusão}

Os resultados desse estudo mostram que os pacientes diabéticos possuem altas taxas de infecção fúngica cutânea, que aparentemente esse perfil não difere dos pacientes residentes em zona urbana. As dermatomicoses mais comuns são as onicomicoses, sendo os dermatófitos os agentes mais frequentes. Além disso, esses resultados sugerem que a predisposição do 
hospedeiro diabético ao desenvolvimento de fungos agentes de dermatomicoses pode estar relacionado à falha na ativação de fagócitos, uma vez que, apesar de existir interação entre o fungo e as células fagocíticas, o mecanismo de morte não foi eficiente, justificando a associação entre DM e infecções fúngicas. Esse assunto é de relevância para a saúde pública, aprimorar conhecimentos sobre a relação fungo/hospedeiro diabético certamente resultará em benefícios ao manejo do paciente diabético e, portanto, será tema de estudos futuros.

\section{Agradecimentos}

Este estudo foi financiado pela Coordenação de Aperfeiçoamento de Pessoal de Nível Superior (CAPES), Conselho Nacional de Desenvolvimento Científico e Tecnológico (CNPq), Fundação de Amparo à Pesquisa do Estado do Paraná (Fundação Araucária) e Financiadora de Estudos e Projetos (FINEP/COMCAP).

\section{Referências}

Akkus, G., Evran, M., Gungor, D., Karakas, M., Sert, M. \& Tetiker, T. (2016). Tinea pedis and onychomycosis frequency in diabetes mellitus patients and diabetic foot ulcers. A cross sectional - observational study. Pak J Med Sci, 32:891-895.

Assadamongkol, R., Lertwattanarak, R., Wannachalee, T., Bunyaratavej, S., Leeyaphan, C. \& Matthapan L. (2016). Prevalence, risk factors, and type of organism in fungal foot infection and toenail onychomycosis in Thai diabetic patients. J Med Assoc Thai, 99:659-664.

Babayani, M., Salari, S., Hashemi, S.J., Ghasemi, N.A.P. \& Fattahi, A. (2018). Onychomycosis due to dermatophytes species in Iran: Prevalence rates, causative agents, predisposing factors and diagnosis based on microscopic morphometric findings. J Mycol Med, 28:45-50.

Basu, S., Yudkin, J.S., Kehlenbrink, S., Davies, J., Wild, S.H., Lipska, K.J., et al. (2018). Estimation of global insulin utilization for Type 2 Diabetes Mellitus, 2018 to 2030: A Microsimulation Model (2018). Lancet Diabetes Endocrinol, 7(1):22-33.

Busui, R., Pop, B.A.J., Feldman, E.L., Bril, V., Freeman, R., Malik, R.A., et al. (2017). Diabetic Neuropathy: A Position Statement by the American Diabetes Association. Diabetes Care, 40:136-154.

Casagranda, L.P., Lange, C., Zillmer, J.G.V., dos Santos F., Castro, D.S.P. \& de Llano, P.M.P. (2018). Prevalência de Diabetes Mellitus em idosos da zona rural no Sul do Brasil. Enfermagem Brasil, 17(4): 09-28.

Cho, N.H., Shaw, J.E., Karuranga, S., Huang, Y., da Rocha Fernandes, J.D, Ohlrogge, A.W. \& Malanda, B. (2018). IDF Diabetes Atlas: Global estimates of diabetes prevalence for 2017 and projections for 2045. Diabetes Res Clin Pract, 138:271-81.

Da Costa, M.L., Moraes, R.B., Vaz, D.W.N., dos Santos, G.A., Duarte, R.C. da C., dos Santos Jr, J.A.B., Menezes, T.X.F. \& Teixeira, R.S. (2021). Avaliação dos pacientes com Diabetes e Hipertensão em uma Estratégia de Saúde da Família localizada na zona rural do interior do Estado do Pará. Res, Soc Dev, 10(3).

Dahlke RJ, Botelho TKR, Loch AP, \& de Cordova, CMM. (2015). Micoses superficiais em pacientes com diabetes mellitus atendidos em um ambulatório geral em Blumenau, SC. Rev Bras Anal Clin, 47:170-3

Da Silva, E.F., Paniz, V.M.V., Laste, G., Torres, I.L. da S. (2013). Prevalência de morbidade e sintomas em idosos: um estudo comparativo entre as áreas rural e urbana. Ciênc. saúde coletiva, 18:1029-40.

Da Silva, R.M.P.R., Nunes e Silva, V.R. \& Paz, F.A. do N. (2021). Ações de enfermagem junto aos portadores de diabetes mellitus residentes da zona rural. Res, Soc Dev, 10(6).

De Zuani, M., Paolicelli, G., Zelante, T., Renga, G., Romani, L., Arzese, A., Pucillo, C.E.M. \& Frossi, B. (2018). Mast cells respond to Candida albicans infections and modulate macrophages phagocytosis of the fungus. Front Immunol, 9: 2829.

Diez-Orejas, R., Feito, M.J., Cicuéndez, M., Casarrubios, L., Rojo, J.M. \& Portolés, M.T. (2018). Graphene oxide nanosheets increase Candida albicans killing by pro-inflammatory and reparative peritoneal macrophages. Biointerfaces Colloids Surf B, 171:250-59.

Dorko, E., Baranová, Z., Jenca, A., Kizek, P., Pilipcinec, E. \& Tkáciková, L. (2005). Diabetes mellitus and candidiases. Folia Microbiol, 50:255-261.

Eba, M., Njunda, A.L., Mouliom, R.N., Kwenti, E.T., Fuh, A.N., Nchanji, G.T. \& Atashili, J. (2016). Onychomycosis in diabetic patients in Fako Division of Cameroon: prevalence, causative agents, associated factors and antifungal sensitivity patterns. BMC Res Notes, 9:1-8.

Erraji, H., Bahtaoui, W., Hali, F., Baline, K., Abdellaoui, M.S., \& Chiheb, S. (2020). Paroniquias chroniques et Candida: étude descriptive et analytique de 164 cases. Ann Dermatol Venereol, 147:143-46.

Foss, N.T., Polon, D.P., Takada, M.H., Foss-Freitas, M.C. \& Foss, M.C. (2005). Dermatoses em pacientes com diabetes mellitus. Rev Saúde Pública, 39:1-5.

Galletti J., Negri, M., Grassi, F.L., Kioshima-Cotica, E.S, \& Svidzinski TIE. (2015). Fusarium spp. is able to grow and invade healthy human nails as a single source of nutrients. Eur J Clin Microbiol Infect Dis, 34:1767-72.

Gross, J.L., Silveiro, S.P., Camargo, J.L., Reichelt, A.J. \& de Azevedo, M.J. (2002). Diabetes Melito: Diagnóstico, Classificação e Avaliação do Controle Glicêmico. Arq Bras Endocrinol Metab, 46:16-26. 
Gupta, A.K., Versteeg, S.G. \& Shear, N.H. (2017). Onychomycosis in the 21st Century: An update on diagnosis, epidemiology, and treatment. J Cutan Med Surg, 21:525-39.

Iser, B.P.M., Stopa, S.R., Chueiri, P.S., Szwarcwald, C.L., Malta, D.C., Monteiro, H.O.C., Duncan, B.B. \& Schmidt, M.I. (2015). Self reported diabetes prevalence in Brazil: results from National Health Survey 2013. Prevalência de diabetes autorreferida en Brasil: resultados de la Encuesta Nacional de Salud 2013. Epidemiol Serv Saúde, 24:305-314.

Larone, D.H. Medically Important Fungi: A Guide to Identification. $5^{\text {th }}$ ed. ASM Press, Washington, DC 2011

Lavery, L.A., Oz, O.K., Bhavan, K. \& Wukich, D.K. (2019). Diabetic Foot Syndrome in the Twenty-First Century. Clin Podiatr Med Surg, 36:355-359.

Lima, A.L., Illing, T., Schliemann, S. \& Elsner, P. (2017). Cutaneous manifestations of Diabetes Mellitus: A Review. Am J Clin Dermatol, 18 : $541-553$.

Lipner, S.R. \& Scher, R.K. (2019). Part I: Onychomycosis: Clinical Overview and Diagnosis. J Am Acad Dermatol, 80: 835-835.

Mader, J.K., Haas, W., Aberer, F., Boulgaropoulos, B., Baumann, P., Pandis, M., Horvath, K., Aziz, K., Köhler, G., Pieber, T.R., Plank, J. \& Sourij, H. (2019). Patients with healed diabetic foot ulcer represent a cohort at highest risk for future fatal events. Sci Rep, 9:10325.

Nenoff, P., Krüger, C., Ginter-Haselmayer, G. \& Tietz, H.J. (2014). Mycology - an update. Part 1: Dermatomycoses: Causative agents, epidemiology and pathogenesis. J Dtsch Dermatol Ges, 12(3);188-210.

Normand, A.C., Becker, P., Gabriel, F., Cassagne, C., Accoceberry. I., Gari-Toussaint, M.L. et al. (2017). Validation of a new web application for identification of fungi by use of matrix-assisted laser desorption ionization-time of flight mass spectrometry. J Clin Microbiol, 55:2661-2670.

Öztürk, A.M., Taşbakan, M., Metin, D.Y., Yener, C., Uysal, S., Şımşır, I.Y., Ertam, I., Pullukçu, H., Arda, B. \& Çetinkalp, S. (2019). A neglected causative agent in diabetic foot infection: a retrospective evaluation of 13 patients with fungal etiology. Turk J Med Sci, 49:81-6.

Oz, Y., Goraan, I., Oz, A. \& Balta, I. (2017). Prevalence and epidemiology of tinea pedis and toenail onychomycosis and antifungal susceptibility of the causative agents in patients with type 2 diabetes in Turkey. Int J Dermatol, 56:68-74.

Pai, Y.W., Lin, C.H., Lee, I.T. \& Chang, M.H. (2018). Prevalence and biochemical risk factors of diabetic peripheral neuropathy with or without neuropathic pain in Taiwanese adults with type 2 diabetes mellitus. Diabetes Metab Syndr, 12:111-116.

Poradzka, A., Jasik, M., Karnafel, W. \& Fiedor, P. (2013). Clinical aspects of fungal infections in diabetes. Acta Pol Pharm, 70:587-596.

Salazar-Tamayo, G., López-Jácome, L.E., Resendiz-Sanchez, J., Franco-Cendejas, R., Rodriguez-Zulueta, P. \& Corzo-León, D. E. (2017). Higher in vitro proliferation rate of Rhizopus oryzae in blood of diabetic individuals in chronic glycaemic control compared with non-diabetic individuals. Mycopathologia, 182:1005-1014.

Sanches, M.M., Roda, A., Pimenta, R., Filipe, P.L., \& Freitas, J.P. (2019). Manifestações cutâneas da diabetes mellitus e pré-diabetes. Acta Med Port. 32:459465 .

Sultana, S., Jaigirdar, Q.H., Islam, M.A. \& Azad, A.K. (2018). Frequency of fungal species of onychomycosis between diabetic and non-diabetic patients. Mymensingh Med J, 27:752-756.

Syzon, O.O., Turkevych, S.A., Rudnyk, T.I., Volbyn, S.V. \& Dashko, M.O. (2019). Superficial candidosis course in patients with carbohydrate metabolism disorder and diabetes mellitus. Wiad Lek, 72:68-71.

Torres, T.B., Izaola, J.O. \& De Luis, R.D.A. (2017). Abordaje nutricional del paciente con diabetes mellitus e insuficiencia renal crónica, a propósito de un caso. Nutr Hosp, 34:18-37.

Varon, A.G., Nouer, S.A., Barreiros, G., Trope, B.M., Magalhães, F., Akiti, T., Garnica, M. \& Nucci, M. (2014). Superficial skin lesions positive for Fusarium are associated with subsequent development of invasive fusariosis. J Infect, 68:85-89.

Veiga, F.F., de Castro-Hoshino, L.V., Sato, F., Bombassaro, A., Vicente, V.A., Mendes, V., Baesso, M.L., Negri, M. \& Svidzinski, T.I.E. (2018). Fusarium oxysporum is an onychomycosis etiopathogenic agent. Future Microbiol, 13:1745-1756.

Xiao, Z., Zhang, X., Geng, L., Zhang, F., Wu, J., Tong, J., Ogunbona, P.O. \& Shan, C. (2017). Automatic non-proliferative diabetic retinopathy screening system based on color fundus image. Biomed Eng Online, ;16:122.

Wang, Y., O’Neil, A., Jiao, Y., Wang, L., Huang, J., Lan, Y., Zhu, Y. \& Yu, C. (2019). Sex differences in the association between diabetes and risk of cardiovascular disease, cancer, and all-cause and cause-specific mortality: a systematic review and meta-analysis of 5,162,654 participants. BMC Med. 17:136. 